\title{
Boosting high-intensity focused ultrasound- induced anti-tumor immunity using a sparse-scan strategy that can more effectively promote dendritic cell maturation
}

\author{
Fang $\mathrm{Liu}^{1 \dagger}$, Zhenlin $\mathrm{Hu}^{1+}$, Lei Qiu', Chun Hui ${ }^{2}$, Chao $\mathrm{Li}^{2}$, Pei Zhong ${ }^{3 *}$, Junping Zhang ${ }^{1 *}$
}

\begin{abstract}
Background: The conventional treatment protocol in high-intensity focused ultrasound (HIFU) therapy utilizes a dense-scan strategy to produce closely packed thermal lesions aiming at eradicating as much tumor mass as possible. However, this strategy is not most effective in terms of inducing a systemic anti-tumor immunity so that it cannot provide efficient micro-metastatic control and long-term tumor resistance. We have previously provided evidence that HIFU may enhance systemic anti-tumor immunity by in situ activation of dendritic cells (DCs) inside HIFU-treated tumor tissue. The present study was conducted to test the feasibility of a sparse-scan strategy to boost HIFU-induced anti-tumor immune response by more effectively promoting DC maturation.
\end{abstract}

Methods: An experimental HIFU system was set up to perform tumor ablation experiments in subcutaneous implanted MC-38 and B16 tumor with dense- or sparse-scan strategy to produce closely-packed or separated thermal lesions. DCs infiltration into HIFU-treated tumor tissues was detected by immunohistochemistry and flow cytometry. DCs maturation was evaluated by IL-12/IL-10 production and CD80/CD86 expression after co-culture with tumor cells treated with different HIFU. HIFU-induced anti-tumor immune response was evaluated by detecting growth-retarding effects on distant re-challenged tumor and tumor-specific IFN- $\gamma$-secreting cells in HIFUtreated mice.

Results: HIFU exposure raised temperature up to 80 degrees centigrade at beam focus within $4 \mathrm{~s}$ in experimental tumors and led to formation of a well-defined thermal lesion. The infiltrated DCs were recruited to the periphery of lesion, where the peak temperature was only 55 degrees centigrade during HIFU exposure. Tumor cells heated to 55 degrees centigrade in 4-s HIFU exposure were more effective to stimulate co-cultured DCs to mature. Sparsescan HIFU, which can reserve 55 degrees-heated tumor cells surrounding the separated lesions, elicited an enhanced anti-tumor immune response than dense-scan HIFU, while their suppressive effects on the treated primary tumor were maintained at the same level. Flow cytometry analysis showed that sparse-scan HIFU was more effective than dense-scan HIFU in enhancing DC infiltration into tumor tissues and promoting their maturation in situ.

Conclusion: Optimizing scan strategy is a feasible way to boost HIFU-induced anti-tumor immunity by more effectively promoting DC maturation.

\footnotetext{
* Correspondence: pzhong@duke.edu; jpzhang08@hotmail.com

† Contributed equally

'Department of Biochemical Pharmacy, School of Pharmacy, Second Military Medical University, Shanghai 200433, China

${ }^{3}$ Department of Mechanical Engineering and Materials Science, Duke University, Box 90300, Durham, NC 27708-0300, USA
} 


\section{Introduction}

In recent years, high-intensity focused ultrasound (HIFU) has emerged as a new and promising treatment modality for a variety of cancers, including breast[1], prostate[2], kidney, liver[3], bone[4], uterus and pancreas cancers $[5,6]$. By focusing acoustic energy in a small cigar-shaped volume inside the tumor, HIFU can rapidly raise the tissue temperature at its beam focus above $65^{\circ} \mathrm{C}$, leading to cellular coagulative necrosis and thermal lesion formation in a well-defined region. In principle, HIFU can be applied to most internal organs with an appropriate acoustic window for ultrasound transmission except those with air-filled viscera such as lung or bowel. In particular, HIFU is advantageous in treating patients with unresectable cancers, such as pancreatic carcinoma, or with poor physical condition for surgery. Unlike radiation and chemotherapy, HIFU can be applied repetitively without the apprehension of accumulating systemic toxicity. This unique feature allows multiple HIFU sessions to be performed if local recurrence occurs. Clinical studies have already demonstrated promising outcome of HIFU treatment for several types of malignances, including prostate cancer, breast cancer, uterine fibroids, hepatocellular carcinomas, and bone malignances $[7,8]$. Although some thermal (skin burn, damage to adjacent bones or nerves) and non-thermal (pain, fever, local infection, and bowel perforation) complications of HIFU treatment have been reported, most of the complications were minor and without severe adverse consequences[8,9].

At present, the primary drawback of HIFU is that it cannot be used to kill micro-metastases outside the primary tumor site. In fact, distant metastasis is a major cause of mortality following clinical HIFU therapy[10]. Lengthy treatment time also represents a limitation. Because each HIFU pulse generally creates an ablated spot of $\sim 10 \times 3 \times 3 \mathrm{~mm}$ in size, up to 1000 lesions may need to be packed closely together during HIFU treatment by scanning the beam focus in a matrix of positions to cover entire tumor volume. With current treatment algorithms, this may translate into a procedure time exceeding 4 hours. Currently, the conventional HIFU treatment protocol in clinic utilizes a dense scanning pattern to eradicate as much tumor mass as possible. Nevertheless, local recurrence of the tumor, due to incomplete tissue necrosis, is still frequently observed following HIFU therapy[10,11]. Clearly, the quality and effectiveness of HIFU cancer therapy need further improvement.

In addition to direct localized destruction of tumor tissues, preliminary evidence from several recent studies has suggested that HIFU may enhance host systemic anti-tumor immunity[12,13]. Although the underlying mechanism is still largely unknown, the potential for a HIFU-elicited anti-tumor immunity is attractive and may help to control local recurrence and distant metastasis following thermal ablation of the primary tumor. On the other hand, the anti-tumor immune response reported in previous studies was not strong enough to achieve a therapeutic gain. As mentioned above, local tumor recurrence and distant metastasis are often the cause of failure for HIFU therapy[10,12], indicating the need to augment the host anti-tumor immunity. Therefore, the optimized strategies that can reduce the primary tumor mass and elicit simultaneously a strong anti-tumor immune response are highly desirable.

The induction and maintenance of an effective antitumor immune response is critically dependent on dendritic cells (DCs), the most effective antigen-presenting cells (APCs) that capture antigens in peripheral tumor tissues and migrate to secondary lymphoid organs, where they cross-present the captured antigens to $\mathrm{T}$ cells and activate them[14]. To act as potent APCs, DCs must undergo maturation, a state characterized by the upregulation of $\mathrm{MHC}$ and costimulatory molecules and the production of cytokines such as IL-12. However, the requisite signals for DC maturation are often absent from the bed of poorly immunogenic tumors, and many tumor cells even actively produce immunosuppressive cytokines such as VEGF to suppress DC function[15]. Thus, DCs infiltrated in tumor tissues typically exhibit a "suppressed" phenotype, and show significantly reduced ability to stimulate allogeneic $\mathrm{T}$ cells when compared with normal DCs. Such alterations in DCs development and function are associated with tumor escape from immune-mediated surveillance[16,17]. On the other hand, several studies have demonstrated that dying tumor cells responding to chemotherapy or radiotherapy can express 'danger' and 'eat me' signals such as heatshock proteins (HSPs) on the cell surface or release intracellular HSP molecules to stimulate DCs to mature and elicit a strong anti-tumor immune response[18]. In the setting of HIFU therapy, we have demonstrated in vitro that HIFU treatment results in the release endogenous immunostimulatory factors from tumor cells and stimulates DCs to mature[19]. We have further provided evidence that HIFU can stimulate DCs to infiltrate into tumor tissues, migrate to draining lymph nodes after being activated, and subsequently elicit tumor-specific CTL responses[20]. Based on these observations, we have postulated that in situ activation of DCs inside HIFU-treated tumor tissue may constitute an important mechanism for HIFU-induced anti-tumor immunity. Given the central role of DCs maturation in the development of anti-tumor immune response, it is reasonable to speculate that an optimized HIFU strategy that can 
more effectively activate DCs to mature should have potential to elicit a stronger anti-tumor immunity.

The present study was conducted to search for a feasible way to boost HIFU-induced anti-tumor immunity by more effectively stimulating DCs to mature. To this end, we set up an experimental HIFU system and performed a series of tumor ablation experiments in subcutaneous implanted MC-38 and B16 tumor models. We found that the infiltrated DCs were mostly recruited to the periphery of thermal lesions after HIFU exposure and the tumor cells at the periphery of HIFU-induced thermal lesions could more effectively stimulated DCs to mature. Based on these finding, we further hypothesize a sparse-scan strategy that can produce separated thermal lesions and reserve surrounding peripheral tumor tissue may provide more stimuli for DC maturation than currently used dense-scan strategy, and finally enhance the strength of HIFU-induced systemic antitumor immune response. By comparing the tumor ablation efficiency and anti-tumor immune response elicited by two different HIFU treatment strategies, i.e., spare vs. dense scan, in well-controlled animal experiments, we demonstrated that it is actually feasible to boost HIFUinduced anti-tumor immunity through optimizing HIFU scan strategy. Finally, we did ex vivo experiments to assess the number of tumor-infiltrating DCs and their maturation status in HIFU-treated tumor tissues and found that sparse-scan HIFU was more effective than dense-scan HIFU in enhancing infiltration of DCs into tumor tissues and promoting their maturation in situ.

\section{Materials and methods Cell culture}

MC-38 mouse colon adenocarcinoma tumor cell line was kindly provided by Dr. Timothy M. Clay of Duke Comprehensive Cancer Center, Duke University (Durham, NC, USA). B16 mouse melanoma cell line and EL4 mouse lymphoma cell line were obtained from Shanghai Institute of Cell Biology and Biochemistry (Shanghai, China). All of cell lines were maintained in complete Dulbeco's modified eagle medium (DMEM), supplemented with $10 \%$ fetal bovine serum (FBS) (Gibco, USA) at $37^{\circ} \mathrm{C}$ and $5 \% \mathrm{CO}_{2}$.

\section{Experimental animals and Tumor Model}

C57BL/6 female mice, 5-8 weeks old, were purchased from Shanghai SLAC Laboratory Animal CO. LTD (Shanghai, China). Tumor models were prepared by subcutaneously injecting $5 \times 10^{5} \mathrm{MC}-38$ or B16 tumor cells suspended in $50 \mu \mathrm{l}$ of PBS in the left hindlimb of the C57BL/6 mice. The tumor was allowed to grow for 8 days to reach a diameter of $8-10 \mathrm{~mm}$ before HIFU treatment. All procedures involving animal treatment and their care in this study were approved by the animal care committee of the
Second Military Medical University in Shanghai in accordance with institutional and Chinese government guidelines for animal experiments.

\section{HIFU Exposure System}

In vivo HIFU treatment of tumor was carried out utilizing a B-mode ultrasound imaging-guided HIFU exposure system as reported in our previous study [20] (Figure 1A). A HIFU transducer (provided by Shanghai A\&S Science Technology Development CO., LTD, Shanghai, China) with a focal length of $63 \mathrm{~mm}$, operated at $3.3 \mathrm{MHz}$ was mounted at the bottom of a tank filled with degassed water. The transducer was driven by sinusoidal signals produced by a function generator connected in series with a 55-dB power amplifier (DF 5857, Ningbo Zhongce Dftek Electronics Co. Ltd, Ningbo, China). The operation and exposure parameters of the HIFU system were controlled by LabView programs via a GPIB board installed in a PC. During the experiment, the anesthetized animal was placed in a customdesigned holder (Figure 1B and 1C) connected to a 3-D positioning system driven by computer-controlled step motors (provided by Shanghai A\&S Science Technology Development CO., LTD, Shanghai, China). To facilitate alignment of the tumor to the HIFU focus, a portable ultrasound imaging system (Terason 2000, Terason, Inc., Burlington, MA) with a $5 / 10 \mathrm{MHz}$ probe was used to provide B-mode images of the tumor cross section. The medial plane of the tumor was aligned with the focus of the HIFU transducer. Figure 1D shows an example of the B-mode ultrasound images of the tumor grown in the hindlimb of the mouse. As shown in the figure, the tumor outline was clearly defined, with the focus of the HIFU transducer highlighted with a cross-hair indicator. Treatment of the tumor was accomplished through progressive scanning of the whole tumor volume point-bypoint, translating the tumor-bearing mouse incrementally with the 3-D step motor positioning system.

In vitro HIFU treatment of tumor cells was performed in a HIFU exposure system shown in Figure 1E. The HIFU transducer was mounted horizontally inside a water tank filled with degassed water. $1 \times 10^{5}$ tumor cells suspended in $20 \mu \mathrm{l}$ DMEM were loaded in a $0.2 \mathrm{ml}$ PCR thin-walled tube, which was placed vertically with its conical bottom aligned within beam focus of the HIFU transducer.

\section{Measurement of temperature profile}

The temperature profile at the HIFU focus was measured by using a Digital Thermometor (MC3000-000, Shanghai DAHUA-CHINO Instrument Co, Ltd, Shanghai, China) with $0.1 \mathrm{~mm}$ bare-wire thermocouple inserted into the tumor tissue or the cell suspension. The thermocouple embedded in the tumor or cell 

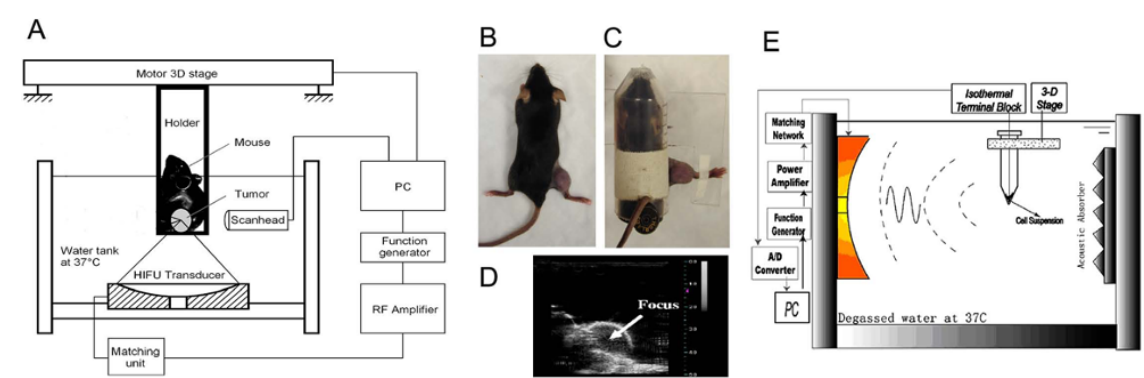

Figure 1 The experimental HIFU system. (A) Diagram of the in vivo HIFU exposure setup. (B) A tumor-bearing mouse. (C) The way the mouse was fixed during HIFU exposure. (D) The B-mode ultrasound image of the tumor. (E) Diagram of the in vitro HIFU exposure setup.

suspension was first aligned to the HIFU focus then temperature elevations and distributions around the center of focus during HIFU exposures were recorded.

\section{Assay of DC infiltration inside tumor tissue by immunohistochemistry}

One day after the HIFU treatment, tumors were surgically excised, freshly frozen in Tissue-Tek O.C.T. compound (Sakura Finetek, Torrance, CA USA), and sectioned at $6 \mu \mathrm{m}$ thickness. The cryostat sections were then fixed in acetone and immunostained with hamster anti-mouse CD11c mAb (clone HL3, PharMingen). Subsequently, the antibody was visualized using an antihamster Ig HRP detection kit (Pharmingen) following the manufacturer's protocol. Finally, sections were counterstained with hematoxylin and evaluated by light microscopy.

\section{Generation of bone marrow-derived DC [19]}

Bone marrow cells were flushed from the femurs and tibiae of female C57BL/6 mice, filtered through a Falcon 100- $\mu \mathrm{m}$ nylon cell strainer (BD Labware), and depleted of red blood cells by five minute incubation in ACK lysis buffer (0.15 M NH4Cl, $1.0 \mathrm{mM}$ KHCO3, $0.1 \mathrm{mM}$ Na2EDTA, pH 7.4). Whole bone marrow cells were plated in six-well plates (BD Labware) in RPMI-1640 supplemented with 10\% FCS (GIBCO-BRL, USA), GM-CSF $(10 \mathrm{ng} / \mathrm{ml})$, and IL-4 (10 ng/ml) (BD Biosciences Pharmingen, USA), and incubated at $37^{\circ} \mathrm{C}$ and $5 \% \mathrm{CO} 2$. Three days later, the floating cells (mostly granulocytes) were removed, and the adherent cells were replenished with fresh medium containing GM-CSF and IL-4. Nonadherent and loosely adherent cells were harvested on day 6 as immature DC (typically contained $>90 \%$ cells expressing CD11c and MHC class II on the surface, as determined by flow cytometry).

\section{In vitro stimulation of DCs with HIFU-treated tumor cells} and assay for their maturation status

$5 \times 10^{5}$ immature DCs generated from mouse bone marrow cells were co-cultured with HIFU-treated B16 tumor cells at ratio of $1: 1$ in $1 \mathrm{ml}$ of culture for 2 days at $37^{\circ} \mathrm{C}$ with $5 \% \mathrm{CO}_{2}$. DC alone, DC stimulated with CpG-ODN1826 (5'-TCCATGACGTTCCTGACGTT-3', Coley Pharmaceutical, Wellesley, MA), which is a known potent DC stimulator, and DC co-cultured with non-HIFU treated B16 tumor cells were used as control. After incubation, supernatants were harvested and assayed for secreted IL-12 and IL-10 by commercial ELISA kits (Biosource International, CA, USA). To analyze the expression levels of co-stimulatory molecules, DCs were collected into cold PBS plus 1\% dialyzed bovine serum albumin, then washed and stained on ice for $30 \mathrm{~min}$ with a combination of the following monoclonal antibodies: FITC-Conjugated Anti-Mouse CD11c, PE-Conjugated Anti-Mouse CD86, and PE-CY5-Conjugated Anti-Mouse CD80 (BD Biosciences Pharmingen, USA). Subsequently, the cells were washed again and analyzed using a FACSCalibur flow cytometer (BectonDickinson).

\section{Tumor growth regression assay}

Following HIFU treatment, Mice were thereafter monitored daily for tumor growth. Mean tumor area for each group was calculated as the product of bisecting tumor diameters obtained from caliper measurements. Measurements were terminated and mice were sacrificed when tumors reached $20 \mathrm{~mm}$ in their largest dimension, or when mice became visibly unwell, or when the tumor became ulcerated.

\section{ELISPOT Assay [20]}

Spleens were harvested from euthanized tumor-bearing mice 14 days after HIFU treatment. Splenocytes from mice bearing MC-38 tumors in each group were restimulated in vitro by culture with mitomycin-pretreated MC-38 (specific) or EL4 (irrelevant) tumor cells at 20:1 responder-to-stimulator ratios for $24 \mathrm{~h}$. Splenocytes from mice bearing B16 tumors were stimulated with 1 $\mu \mathrm{g} / \mathrm{ml}$ of relevant peptides mouse TRP2 $2181-188$ (VYDFFVWL, purchased from Dalton Chemical Laboratories Inc. Toronto, ON, Canada), or irrelevant control 
peptide $\left(\mathrm{OVA}_{257-264}\right.$ : SIINFEKL) for $24 \mathrm{~h}$. Re-stimulated splenocytes $\left(1 \times 10^{6}\right.$ cells in $100 \mu$ medium $)$ were then plated in 96-well nitrocellulose filter plates pre-coated with anti-mouse interferon- $\gamma$ antibody (Pharmingen, San Diego, CA). After incubation for $24 \mathrm{~h}$ at $37^{\circ} \mathrm{C}$ and $5 \%$ $\mathrm{CO} 2$, the plates were washed with PBS, and "spots," corresponding to cytokine-producing cells, were visualized by incubation with $100 \mu \mathrm{l}$ per well of biotinylated antimouse IFN- $\gamma \mathrm{Ab}$ (Pharmingen) overnight at $4^{\circ} \mathrm{C}$. After washing with PBS $/ 0.5 \%$ Tween, $1.25 \mu \mathrm{g} / \mathrm{ml}$ avidin alkaline phosphatase (Sigma) was added to the well in 100 $\mu \mathrm{l}$ PBS for 1 hour at room temperature. The development of the assay was then performed with $100 \mu \mathrm{l}$ of 5 bromo-4-chloro-3-indolylphosphate/nitro blue tetrazolium (BCIP/NBT tablets, Sigma) for 10 minutes. The reaction is stopped by the addition of water and the plates allowed drying before counting individual spots with a Zeiss automated ELISPOT reader. The results were expressed as the number of spot-forming cells per $10^{6}$ input cells. Overall, three independent experiments were performed with six replicate wells included in each treatment.

\section{Assay of DC infiltration inside tumor tissue by flow cytometry}

One day after the HIFU treatment, tumors were surgically excised. Single cell suspensions were generated from resected tumors as previously described[21]. Briefly, tumors were diced in $\mathrm{Ca}^{2+}$ - and $\mathrm{Mg}^{2+}$-free HBSS after resection, and incubated with $1 \mathrm{mg} / \mathrm{ml}$ type IV collagenase (Sigma-Aldrich) for $90 \mathrm{~min}$ at room temperature and under constant stirring. EDTA (2 mM) was added to the mixture for 30 additional min before filtration of the cell suspension on 70- $\mu$ m cell strainers (BD Biosciences). The cell suspension was finally washed twice in HBSS before analysis. For flow cytometry, the following fluorochrome-conjugated antibodies (all purchased from BD PharMingen) were used for staining: CD45-FITC, CD11C-PE, I-A-PE-CY5, CD80-PE-CY5, CD-80-PE-CY5. After adding the appropriate antibody, the cells were incubated at $4^{\circ} \mathrm{C}$ for 30 min in PBS plus $1 \%$ of dialyzed bovine serum albumin and washed twice by centrifugation using fluorescence-activated cell sorting (FACS) buffer. Fluorescence was analyzed with a FACSCalibur flow cytometer and the CellQuest software (Becton-Dickinson).

\section{Results and Discussion}

\section{HIFU system could produce a typical thermal effect on} experimental tumors

In clinical HIFU therapy, tumor tissue was ablated predominantly by thermal effect which is dependent on the temperature elevation achieved at beam focus during HIFU exposure. If the temperature is raised to $56^{\circ} \mathrm{C}$ or higher in the tissue, thermal lesion will form within a few seconds as a result of cellular coagulative necrosis. In fact, the temperature within the focal volume may rise rapidly above $80^{\circ} \mathrm{C}$ during HIFU treatments[22]. In the present study, we at first calibrated our HIFU system to achieve a typical thermal effect on experimental tumors. By adjusting output pressure level and exposure duration, we found that, when the transducer was run in continuous wave $(\mathrm{CW})$ mode at a pressure level of $\mathrm{P}^{+}$ $=19.5 / \mathrm{P}^{-}=-7.2(\mathrm{MPa})$, an elevated temperature was achieved up to $80^{\circ} \mathrm{C}$ within $4 \mathrm{~s}$ at the beam focus in both MC-38 and B16 tumor (Figure 2A). This temperature profile is a representative of the clinical HIFU dosage used in cancer therapy. Under this condition, one HIFU exposure could generate a typical thermal lesion with a well-defined size of $1 \times 5 \mathrm{~mm}$ (transverse $\times$ longitudinal direction) in the treatment region (Figure $2 \mathrm{C}$ and $2 \mathrm{D}$ ). The peripheral tissue around thermal lesion was also heated but with a lower peak temperature (around $55^{\circ} \mathrm{C}$ ) (Figure $2 \mathrm{~B}$ ).

\section{The infiltrated DCs were mostly recruited to the} periphery of thermal lesions after hifu exposure

We next investigated whether HIFU can enhance infiltration of DCs into treated tumor tissues. Tumor samples were collected 1 day after HIFU treatment, and 6$\mu \mathrm{m}$ cryostat sections were cut and stained with antiCD11c Abs. Figure 3 showed the results of a representative experiment. In the untreated tumor, only a small amount of DC infiltration was observed. In contrast, DC infiltration was enhanced in HIFU-treated tumor tissues. Most interestingly, it was noted that the infiltrated DC was recruited to the periphery of thermal lesion (Figure 3).

\section{Tumor cells at the periphery of HIFU-Induced thermal lesion may possess a stronger immunostimulatory property for DCs maturation}

A prior study has documented a significant up-regulation of HSPs at the border zone of HIFU-induced thermal lesion in patients with benign prostatic hyperplasia [23]. HSPs have been shown to interact with a number of receptors present on the surface of DCs and promote their maturation[24]. These findings imply the possibility that tumor cells at the periphery of HIFU-induced thermal lesion may possess a stronger immunosimulatory property for DCs maturation. The finding in this study that the infiltrated DCs were mostly recruited to the periphery of thermal lesions after HIFU exposure further raises the possibility that tumor cells within this specific zone may have distinct impacts on infiltrated DCs. To provide experimental evidence, we co-cultured immature DCs generated from mouse bone marrow cells with different HIFU-treated tumor cells and 

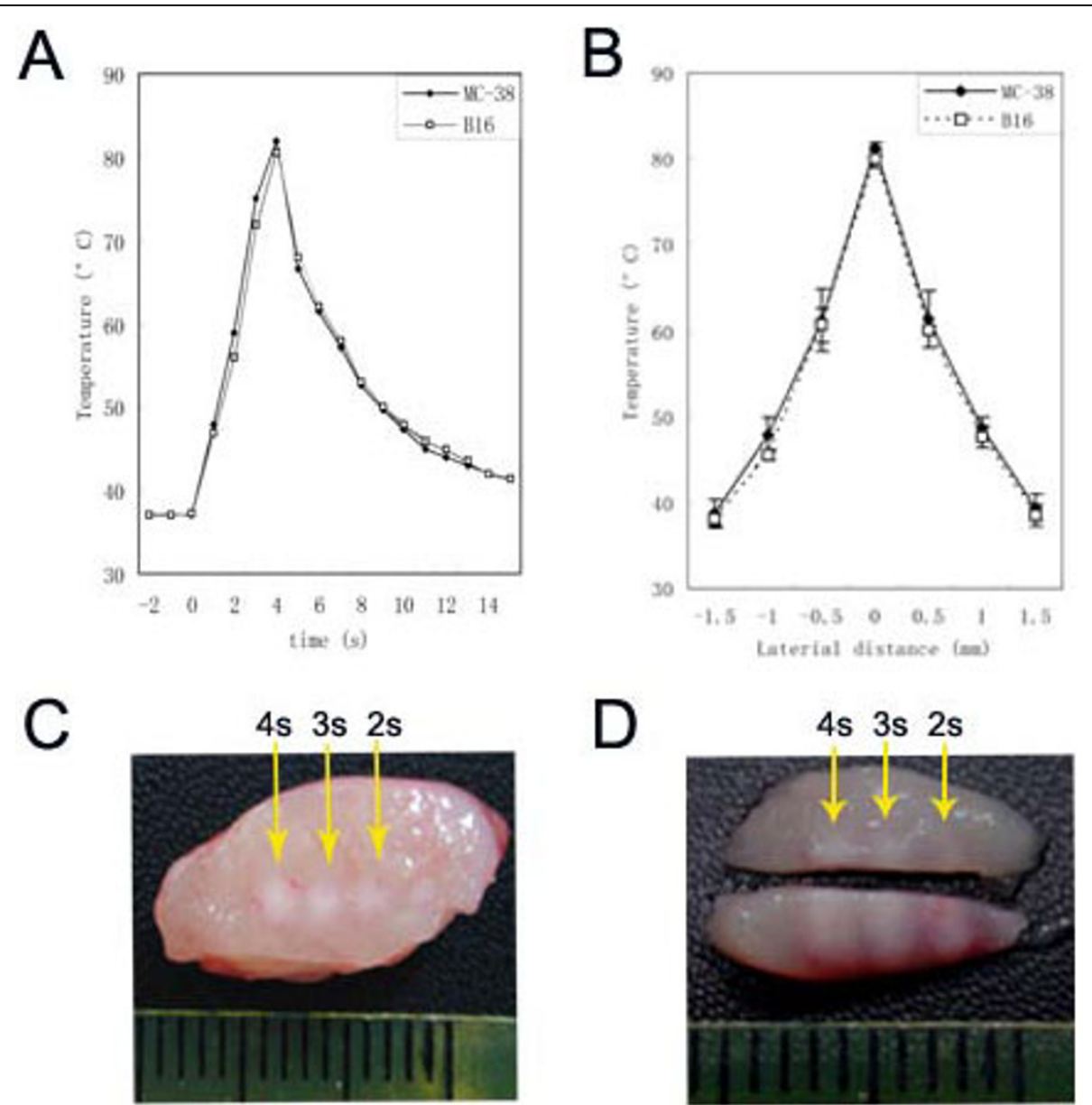

Figure 2 Thermal effects of HIFU treatment. (A) Temperature profiles at the beam focus in MC-38 and B16 tumors when the transducer was run in continuous wave (CW) mode at a pressure level of $\mathrm{P}^{+}=19.5 / \mathrm{P}^{-}=-7.2 \mathrm{MPa}$. Representative data of three independent experiments with consistent results are shown. (B) Lateral distribution of peak temperature in tumors produced by HIFU during 4-s exposures. Results are expressed as means \pm SD out of four independent experiments. (C) Transversal and (D) longitudinal views of thermal lesions produced by HIFU with different treatment duration $(4,3$, and $2 \mathrm{~s})$ at above pressure level. The representative section from four treated mice with similar results is shown.

assessed their maturing status by assay of IL-12p70/IL10 production and CD80/86 expression on DCs. We at first determined two different in vitro HIFU exposure conditions, under which the temperature in the cell suspension could reach a peak value of $55^{\circ} \mathrm{C}$ and $80^{\circ} \mathrm{C}$ respectively within a 4-s exposure duration. Figure $4 \mathrm{~A}$ showed the distinct temperature profiles in tumor cell suspensions produced by the two different HIFU exposure conditions, which correspond to those produced in vivo by HIFU at the periphery and the center of thermal lesion, respectively. For convenience, these exposure conditions were referred to hereafter as " $55^{\circ} \mathrm{C}$-HIFU" and " $80^{\circ} \mathrm{C}$-HIFU", respectively. After HIFU treatment, B16 tumor cells were co-culture with immature DC for 2 days, and the release of IL-12p70 and IL-10 and surface expression of maturation markers (CD80 and CD86) on DCs were assayed. DC alone, DC stimulated with CpG-ODN, and DC co-cultured with non-HIFU treated B16 tumor cells were used as control. The results were shown in figure 4B-D. DCs did not spontaneously secrete IL-12p70 and IL-10 when cultured in the absence of exogenous stimuli. CpG-ODN, a known potent DC stimulator, induced the highest level of IL12p70 production while only moderately increasing IL10 production, and significantly enhanced the expression of CD80 and CD86, indicating CpG-ODN induced immature DC towards a mature phenotype. Normal B16 tumor cells shown no effects on IL-12 p70 production but markedly increased IL-10 production, and significantly decreased the expressions of CD80 and CD86. Since IL-12p70 and IL-10 are reported as immunostimulatory versus immunosuppressive DC-produced cytokines that may differentially affect the functional outcome of T-cell cross-priming $[25,26]$, this result 


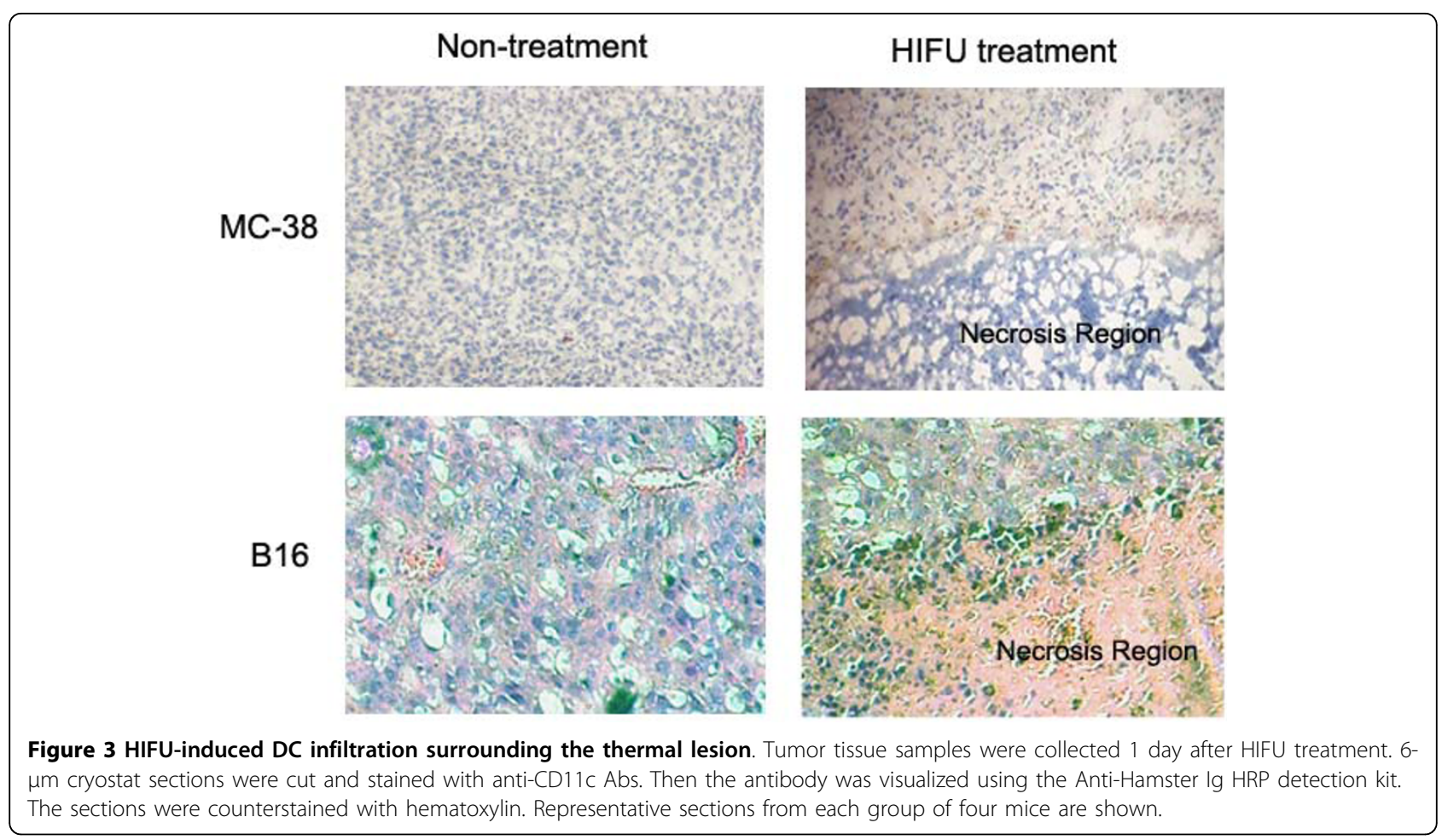

confirmed previous finding that normal tumors could induce or restrict tumor-infiltrating DCs towards an immature phenotype [16,27]. After HIFU-treatment, however, tumor cells became effective in inducing IL12 p70 production while their effects on IL-10 production markedly reduced. Furthermore, both $55^{\circ} \mathrm{C}$-HIFUand $80^{\circ} \mathrm{C}$-HIFU-treated tumor cells significantly enhanced surface expressions of CD80 and CD86 on cocultured DCs. More importantly, $55^{\circ} \mathrm{C}$-HIFU-treated tumor cells showed much more potent immunostimulatory activities than $80^{\circ} \mathrm{C}$-HIFU-treated ones, both in the induction of IL-12p70 production and in the upregulation of CD80 and CD86 expression.

Similar results were obtained with the other cell line MC-38 (data not shown). These results demonstrated that HIFU-treatment can change tumor cells from immunosuppressive to immunostimulatory for DCs maturation. More importantly, tumor cells exposed to ' $55^{\circ} \mathrm{C}$-HIFU', which produced a temperature elevation similar to that at the periphery of thermal lesion, exhibited a markedly stronger immunostimulatory potency than those exposed to ' $80^{\circ} \mathrm{C}$-HIFU', which produced a temperature elevation similar to that at the center of thermal lesion. These data therefore provide evidence that tumor cells at the periphery of thermal lesions can more effectively activate DCs to mature than those within the lesions.

We speculated that intracellular HSP molecules release or their membrane exposure induced by HIFU treatments may be the keynote mechanism responsible for the stimulatory activities of DC maturation provided by HIFU-treated tumor cells. We have done some pilot experiments to compare the effects of different HIFU treatments on the expression of HSPs in tumor cells. Our preliminary results suggested the HIFU treatments caused significant up-regulations of HSP70 and HSP90 expression in tumor cells, in which $55^{\circ} \mathrm{C}$-HIFU was more effective than $80^{\circ} \mathrm{C}$-HIFU (Data not shown). Further studies are underway to determine whether these up-regulated HSPs are released in the extracellular milieu or translocated to cell surface to investigate more deeply the mechanisms of DC activation by HIFU-treated tumor cells.

\section{It is feasible to boost HIFU-induced anti-tumor immunity through optimizing scan strategy}

A dense-scan strategy is usually used in clinical HIFU therapy to produce closely packed or even overlapped thermal lesions to achieve a complete tumor ablation because tumor cells at the board zone of thermal lesion are used to be considered to be heated only sub-lethally and may survive HIFU treatment. However, our data suggest that the presence of such cells in situ may lead to "clinical benefit' by potently activating infiltrated DCs to mature. Since the maturation of tumor-infiltrating DCs will lead to the development of strong anti-tumor immunity, an optimized strategy that can reserve these cells in HIFU-treated tumor may have a potential to 


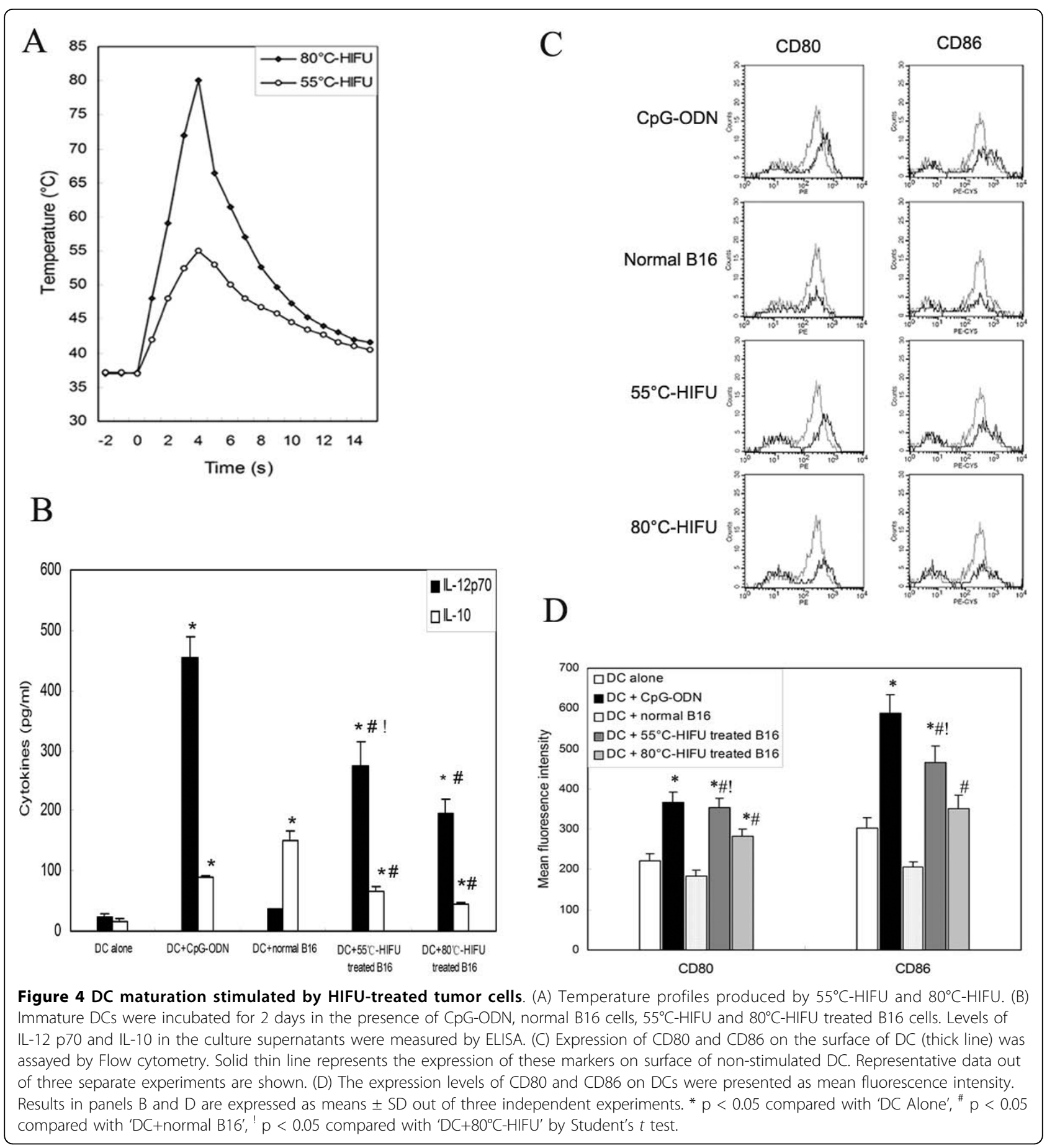

elicit a stronger anti-tumor immune response. In the clinical setting, the simplest way to achieve this goal is to adjusting the scan strategy, e.g. using a sparse-scan strategy to produce separated rather than closely packed thermal lesions. Hence, we further proposed a sparsescan strategy may elicit a stronger systemic anti-tumor immune response than currently used dense-scan strategy. To test this hypothesis, we compared the tumor ablation efficiency and anti-tumor immune response elicited by two different HIFU treatment strategies, i.e., sparse vs. dense scan, in well-controlled animal experiments. Because our HIFU system can produced a thermal lesion with a well define size of $1 \times 5 \mathrm{~mm}$ in the experimental tumor by one pulse of HIFU exposure, a step size of $1 \mathrm{~mm}$ was used in dense-scan strategy which can produce closely packed thermal lesions and 
well mimic the conventional treatment protocol in clinical HIFU therapy. In sparse-scan strategy, the step size was increased to $2 \mathrm{~mm}$ to produce a cluster of separated lesions with inter-lesion spacing of $1 \mathrm{~mm}$. Figure 5A showed the closely packed and separated lesions in MC38 tumor produced by the dense- and sparse-scan strategy, respectively. Tumor growth regression assay revealed HIFU treatment with the sparse- and densescan strategies have similar retarding effects on growth of treated tumors (Figure $5 \mathrm{~B}$ and $5 \mathrm{C}$ ), even though the total number of thermal lesions produced by sparse scan strategy is much less than that in dense scan strategy. To further assess whether HIFU treatments could induce a systemic anti-tumor immune response in vivo, tumor challenge experiments were performed one day following HIFU treatment by injecting $1 \times 10^{6} \mathrm{MC}-38$ or B16 cells subcutaneously in the contra lateral hindlimb. As expected, the sparse-scan HIFU was found to have a stronger retarding effect on challenged tumor growth (Figure 5D and 5E). To further quantify the anti-tumor immune response, we evaluated whether HIFU treatment could elicit tumor-specific IFN- $\gamma$-secreting cells using ELISPOT assay. Consistent with finding in tumor challenge experiments, splenocytes retrieved on day 14 after tumor inoculation in HIFU-treated mice contained significantly more tumor-specific IFN- $\gamma$ secreting cells than that from the control group (Figure $5 \mathrm{~F}$ and 5G). Taken all together, these results demonstrated that optimization of scan strategy in HIFU treatment can indeed induce a more powerful anti-tumor effect and immune response. Here we only focused on proof of principle, so we did not further optimize the inter-lesion spacing or the total number of lesions for the most effective treatment outcome in the present study.

\section{Sparse-scan HIFU was more effective than dense-scan HIFU in enhancing infiltration of DCs into tumor tissues and promoting their maturation in situ}

In order to provide more experimental evidence that the boosted antitumor immune response by sparse-scan HIFU is associated with the stage of the maturation of DCs recruited to the treated tumor, we next determined whether different HIFU treatment could differentially alter DC numbers in the tumor tissues and their functional status. We treated C57BL/6 mice bearing B16 or MC-38 tumors in the left hindlimb with HIFU under sparse- or dense-scan strategy. On the day following HIFU treatment, mice were sacrificed. Upon tumor dissociation, single cell suspensions were generated from

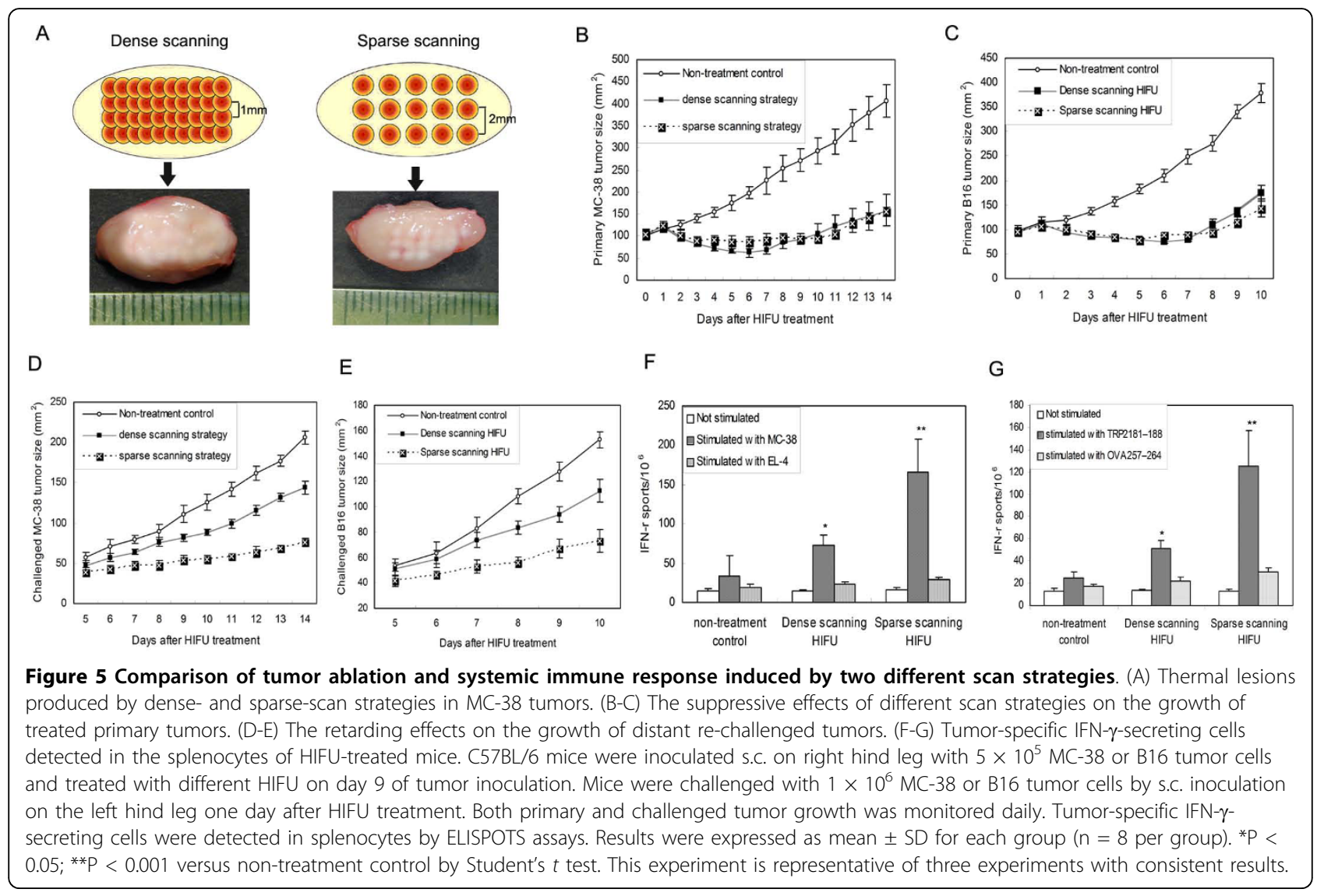


resected tumors. The presence of cells with a DC phenotype and their surface expression of the activation markers MHC class II (MHC II), CD80, and CD86 were analyzed by flow cytometry after immunostainning. Leukocytic cells $\left(\mathrm{CD} 45^{+}\right)$could be distinguished by FACS analysis from malignant cells by their size (FSC-H) and morphology (SSC-H) (Figure 6A). In the leukocytic cell population, $\mathrm{CD} 11 \mathrm{c}^{+} / \mathrm{MHC} \mathrm{II}^{+}, \mathrm{CD} 11 \mathrm{c}^{+} / \mathrm{CD}^{+} 6^{+}$, and $\mathrm{CD} 11 \mathrm{c}^{+} / \mathrm{CD} 80^{+}$cells were visualized (Figure $6 \mathrm{~B}$ ), indicating the presence of cells with a DC phenotype. Notably, higher proportion of tumor-infiltrating DCs (CD11c $+/ \mathrm{MHC} \mathrm{II}^{+}$cells) were recovered from HIFU-treated tumors, as compared with non-treatment control (Figure $6 \mathrm{C}$ and $6 \mathrm{~B}$ ), indicating that HIFU-treatment enhanced DC infiltration into tumor tissues. Furthermore, upregulated levels of the maturation markers CD80 and CD86 were found in CD11c+ subpopulation recovered from HIFU-treated tumors (Figure 6B, D, and 6E), indicating HIFU-treatment induced infiltrated DCs towards a mature phenotype. Most importantly, Sparse-scan HIFU was found to be more effective than dense-scan HIFU in enhancing infiltration of DCs into tumor tissues and promoting their maturation in situ, as evidenced by higher proportion of tumor-infiltrating DCs

A

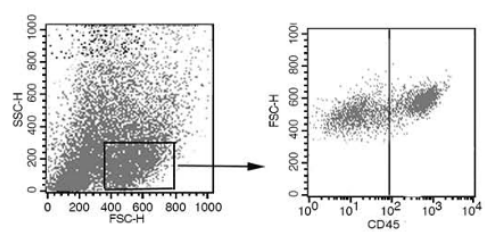

B
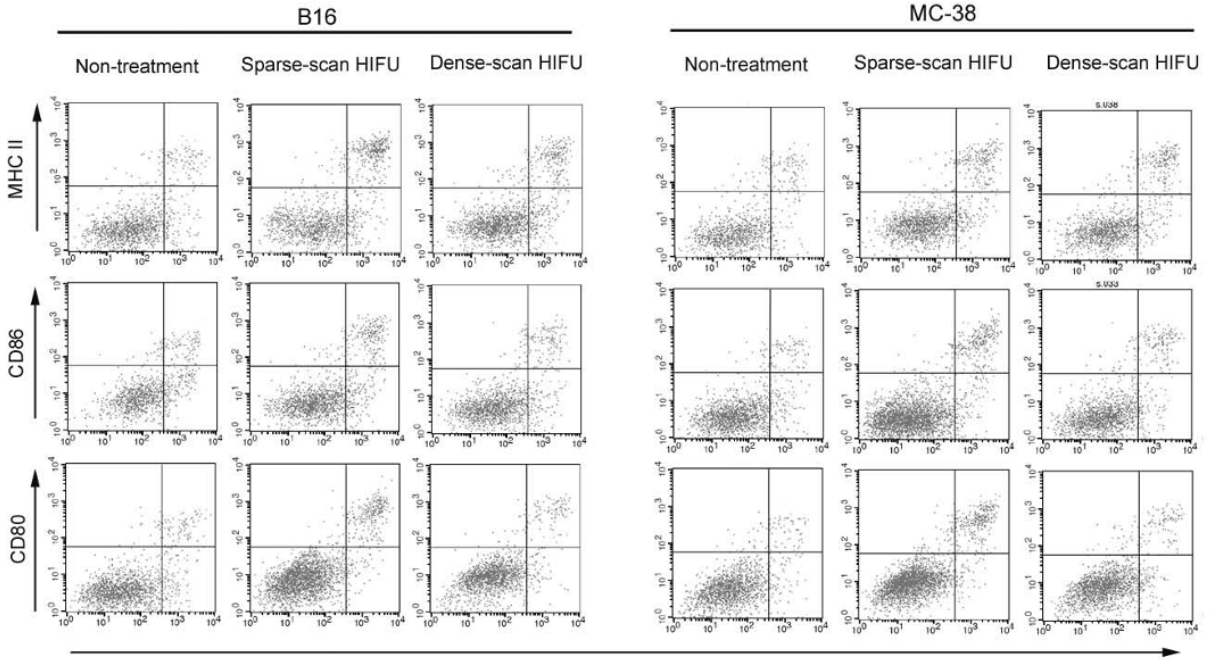

CD11c

C

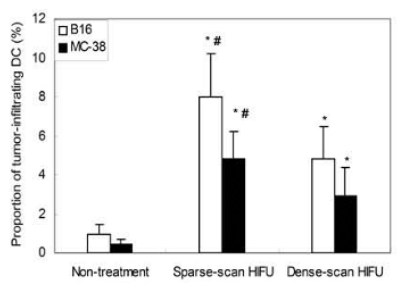

D

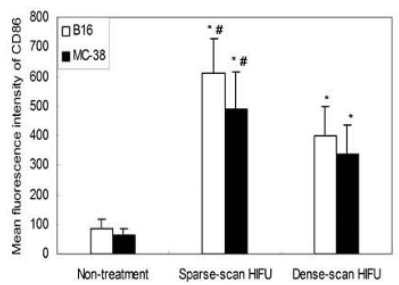

E

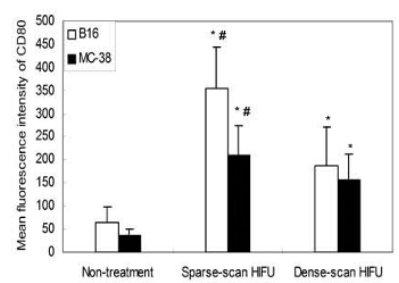

Figure 6 DCs were recruited into tumor tissues one day after HIFU treatment and exhibited the surface phenotype of maturation. (A) The presence of $\mathrm{CD}_{4} 5^{+}$tumor-infiltrating leukocytes in tumor tissues was identified in the gate indicated. (B) CD11 $\mathrm{C}^{+}$cells in the gate defined in A were analyzed for the expression of $\mathrm{MHC} \mathrm{II,} \mathrm{CD80,} \mathrm{and} \mathrm{CD86.} \mathrm{Representative} \mathrm{data} \mathrm{of} \mathrm{six} \mathrm{independent} \mathrm{experiments} \mathrm{with} \mathrm{consistent} \mathrm{results} \mathrm{are}$ shown. (C) The proportion of tumor-infiltrating $D C\left(C D 11 \mathrm{C}^{+} / \mathrm{MHC} \|^{+}\right)$(expressed in percentage of total cells) was investigated for the indicated tumors one day after different HIFU-treatment. (D) The expression levels of CD86 (presented as mean fluorescence intensity) were analyzed in CD11c+ cells infiltrating B16 or MC-38 tumor one day after different HIFU-treatment. (E) The expression levels of CD80 (presented as mean fluorescence intensity) were analyzed in CD11 ${ }^{+}$cells infiltrating B16 or MC-38 tumor one day after different HIFU-treatment. (C-E) Results were expressed as mean \pm SD for each group ( $n=6$ per group). ${ }^{*} \mathrm{P}<0.05$ versus non-treatment control; ${ }^{\#} \mathrm{P}<0.05$ versus Dense-scan HIFU by Student's $t$ test. This experiment is representative of three experiments with consistent results. 
and their higher levels of surface maturation markers (Figure 6B-E). These results suggest that the enhanced antitumor immune response induced by sparse-scan HIFU may be associated with more DC infiltration into treated tumors and effective DC maturation in situ.

\section{Conclusion}

In the present study, our results showed that a sparsescan strategy in HIFU ablation of tumor produced separated thermal lesions with a proper intra-lesion space and elicited a stronger systemic anti-tumor immune response than currently used dense-scan HIFU strategy, which is associated with more effective promotion of DC maturation by tumor cells at the periphery of thermal lesions.

These preliminary findings have significant implications for improving HIFU treatment of cancer in clinic. The conventional treatment protocol in clinical HIFU therapy utilizes a dense-scan strategy to produce closely packed or overlapped thermal lesions aiming at eradicating as much tumor mass as possible. Although effective in reducing the primary tumor mass, this strategy is time consuming and is not most effective in terms of induction of systemic anti-tumor immunity, so that it can not provide efficient micro-metastatic control and long-term tumor resistance for cancer patients. Here we proposed that, by simply adjusting scan strategy to produce separated thermal lesions with a proper intralesion space, a stronger systemic anti-tumor immune response may be elicited while not impair its tumor ablation efficiency, so that the overall quality and effectiveness of HIFU cancer therapy can be improved and the treatment time can be significantly shorten. Future clinical studies will tell whether this promise comes true.

\section{Acknowledgements}

This work was supported in part by National Natural Science Foundation of China No 30772072 and Shanghai Pujiang Program No 08PJ1400200.

\section{Author details}

${ }^{1}$ Department of Biochemical Pharmacy, School of Pharmacy, Second Military Medical University, Shanghai 200433, China. ${ }^{2}$ School of Life Sciences \& Biotechnology, Shanghai Jiao Tong University, China. ${ }^{3}$ Department of Mechanical Engineering and Materials Science, Duke University, Box 90300, Durham, NC 27708-0300, USA.

\section{Authors' contributions}

$\mathrm{FL}$ and $\mathrm{ZH}$ conducted the study, participated in data interpretation, performed the statistical analysis, and drafted the manuscript. LQ participated in the in vitro studies. $\mathrm{CH}$ and $\mathrm{CL}$ participated in the in vivo studies. PZ and JP participated in design, coordination, and data interpretation and drafted the manuscript. All authors read and approved the final manuscript.

\section{Competing interests}

The authors declare that they have no competing interests.

Received: 29 September 2009

Accepted: 27 January 2010 Published: 27 January 2010
References

1. Hynynen K, Pomeroy O, Smith DN, Huber PE, McDannold NJ, Kettenbach J, Baum J, Singer S, Jolesz FA: MR imaging-guided focused ultrasound surgery of fibroadenomas in the breast: a feasibility study. Radiology 2001, 219:176-185.

2. Chaussy C, Thuroff S: The status of high-intensity focused ultrasound in the treatment of localized prostate cancer and the impact of a combined resection. Curr Urol Rep 2003, 4:248-252.

3. Kennedy JE, Wu F, ter Haar GR, Gleeson FV, Phillips RR, Middleton MR, Cranston D: High-intensity focused ultrasound for the treatment of liver tumours. Ultrasonics 2004, 42:931-935.

4. Chen W, Wang Z, Wu F, Zhu H, Zou J, Bai J, Li K, Xie F: [High intensity focused ultrasound in the treatment of primary malignant bone tumor]. Zhonghua Zhong Liu Za Zhi 2002, 24:612-615.

5. Zhang L, Chen WZ, Liu YJ, Hu X, Zhou K, Chen L, Peng S, Zhu H, Zou HL, Bai J, Wang ZB: Feasibility of magnetic resonance imaging-guided high intensity focused ultrasound therapy for ablating uterine fibroids in patients with bowel lies anterior to uterus. Eur J Radiol 2008.

6. Xiong LL, Hwang JH, Huang XB, Yao SS, He CJ, Ge XH, Ge HY, Wang XF: Early clinical experience using high intensity focused ultrasound for palliation of inoperable pancreatic cancer. Jop 2009, 10:123-129.

7. Thuroff S, Chaussy C, Vallancien G, Wieland W, Kiel HJ, Le Duc A, Desgrandchamps F, De La Rosette JJ, Gelet A: High-intensity focused ultrasound and localized prostate cancer: efficacy results from the European multicentric study. J Endourol 2003, 17:673-677.

8. Wu F, Wang ZB, Chen WZ, Wang W, Gui Y, Zhang M, Zheng G, Zhou Y, $\mathrm{Xu} \mathrm{G}, \mathrm{Li} \mathrm{M}$, et al: Extracorporeal high intensity focused ultrasound ablation in the treatment of 1038 patients with solid carcinomas in China: an overview. Ultrason Sonochem 2004, 11:149-154.

9. Visioli AG, Rivens IH, ter Haar GR, Horwich A, Huddart RA, Moskovic E, Padhani A, Glees J: Preliminary results of a phase I dose escalation clinical trial using focused ultrasound in the treatment of localised tumours. Eur J Ultrasound 1999, 9:11-18.

10. Wu F, Wang ZB, Chen WZ, Zou JZ, Bai J, Zhu H, Li KQ, Xie FL, Jin CB, Su HB, Gao GW: Extracorporeal focused ultrasound surgery for treatment of human solid carcinomas: early Chinese clinical experience. Ultrasound Med Biol 2004, 30:245-260.

11. Van Leenders GJ, Beerlage HP, Ruijter ET, de la Rosette JJ, Kaa van de CA: Histopathological changes associated with high intensity focused ultrasound (HIFU) treatment for localised adenocarcinoma of the prostate. J Clin Pathol 2000, 53:391-394.

12. Wu F, Wang ZB, Lu P, Xu ZL, Chen WZ, Zhu H, Jin CB: Activated antitumor immunity in cancer patients after high intensity focused ultrasound ablation. Ultrasound Med Biol 2004, 30:1217-1222.

13. Zhou Q, Zhu XQ, Zhang J, Xu ZL, Lu P, Wu F: Changes in circulating immunosuppressive cytokine levels of cancer patients after high intensity focused ultrasound treatment. Ultrasound Med Biol 2008, 34:81-87.

14. Banchereau J, Steinman RM: Dendritic cells and the control of immunity. Nature 1998, 392:245-252.

15. Takahashi A, Kono K, Ichihara F, Sugai H, Fujii H, Matsumoto Y: Vascular endothelial growth factor inhibits maturation of dendritic cells induced by lipopolysaccharide, but not by proinflammatory cytokines. Cancer Immunol Immunother 2004, 53:543-550.

16. Almand B, Clark JI, Nikitina E, van Beynen J, English NR, Knight SC, Carbone DP, Gabrilovich DI: Increased production of immature myeloid cells in cancer patients: a mechanism of immunosuppression in cancer. J Immunol 2001, 166:678-689.

17. Satthaporn S, Robins A, Vassanasiri W, El-Sheemy M, Jibril JA, Clark D, Valerio D, Eremin O: Dendritic cells are dysfunctional in patients with operable breast cancer. Cancer Immunol Immunother 2004, 53:510-518.

18. Tesniere A, Panaretakis T, Kepp O, Apetoh L, Ghiringhelli F, Zitvogel L, Kroemer G: Molecular characteristics of immunogenic cancer cell death. Cell Death Differ 2008, 15:3-12.

19. Hu Z, Yang XY, Liu Y, Morse MA, Lyerly HK, Clay TM, Zhong P: Release of endogenous danger signals from HIFU-treated tumor cells and their stimulatory effects on APCs. Biochem Biophys Res Commun 2005, 335:124-131.

20. Hu Z, Yang XY, Liu Y, Sankin GN, Pua EC, Morse MA, Lyerly HK, Clay TM, Zhong P: Investigation of HIFU-induced anti-tumor immunity in a murine tumor model. J Transl Med 2007, 5:34. 
21. Preynat-Seauve $O$, Schuler P, Contassot E, Beermann F, Huard B, French LE: Tumor-infiltrating dendritic cells are potent antigen-presenting cells able to activate T cells and mediate tumor rejection. J Immunol 2006, 176:61-67.

22. Hill CR, Rivens I, Vaughan MG, ter Haar GR: Lesion development in focused ultrasound surgery: a general model. Ultrasound Med Biol 1994, 20:259-269.

23. Kramer G, Steiner GE, Grobl M, Hrachowitz K, Reithmayr F, Paucz L, Newman M, Madersbacher S, Gruber D, Susani M, Marberger M: Response to sublethal heat treatment of prostatic tumor cells and of prostatic tumor infiltrating T-cells. Prostate 2004, 58:109-120.

24. Tsan MF, Gao B: Heat shock proteins and immune system. J Leukoc Biol 2009, 85:905-910.

25. Trinchieri G: Interleukin-12 and the regulation of innate resistance and adaptive immunity. Nat Rev Immunol 2003, 3:133-146.

26. Vicari AP, Trinchieri G: Interleukin-10 in viral diseases and cancer: exiting the labyrinth. Immunol Rev 2004, 202:223-236.

27. Gabrilovich DI, Ciernik IF, Carbone DP: Dendritic cells in antitumor immune responses. I. Defective antigen presentation in tumor-bearing hosts. Cell Immunol 1996, 170:101-110.

doi:10.1186/1479-5876-8-7

Cite this article as: Liu et al:: Boosting high-intensity focused

ultrasound-induced anti-tumor immunity using a sparse-scan strategy

that can more effectively promote dendritic cell maturation. Journal of

Translational Medicine 2010 8:7.

\section{Submit your next manuscript to BioMed Central} and take full advantage of:

- Convenient online submission

- Thorough peer review

- No space constraints or color figure charges

- Immediate publication on acceptance

- Inclusion in PubMed, CAS, Scopus and Google Scholar

- Research which is freely available for redistribution

Submit your manuscript at www.biomedcentral.com/submit
C Biomed Central 